Scientific Visualization, 2019, volume 11, number 5, pages 83 - 100, DOI: 10.26583/sv.11.5.08

\title{
Computer visualization in the course "Abstract algebra"
}

\author{
V.I. Varankinaํ, E.N. Lubyagina², R.V. Markov3, A.A. Petrov4, D.V. Shirokov5 \\ Vyatka State University, Department of Fundamental Mathematics, Kirov \\ ${ }^{1}$ ORCID: 0ooo-0o03-4166-1182, veravarankina@gmail.com \\ 2 ORCID: 0000-0001-5071-6208, shishkina.en@mail.ru \\ 3 ORCID: 0000-0002-6560-0483, markovrv@yandex.ru \\ 4 ORCID: oooo-0002-5877-2850, andreipetrow@mail.ru \\ 5 ORCID: 0000-0002-9465-4851, dimshirokov79@mail.ru
}

\begin{abstract}
The work presents the experience of introducing visualization in the teaching of the discipline "Abstract Algebra" to bachelor students specializing in Mathematics. We propose to apply visualization elements at different stages of training: in presenting theoretical information, in solving practical problems, in the process of control and self-control. The article gives an example of using the GeoGebra system to create visualization components. Here we propose the option of introducing the created objects into educational presentations with the help of LaTex computer imposition system. We used Javascript and PHP programming languages, MySQL databases, and the APACHE WEB server to create a course site and to incorporate interactive elements into it.
\end{abstract}

Keywords: abstract algebra, visualization, computer algebra systems, computer calculations, virtuallab, GeoGebra, LaTex, Javascript and PHP, MySQL DB.

\section{Introduction}

In this article, as in [1], by computer visualization of mathematical concepts, principles, problems or processes, we mean their geometric or graphical representations created using the computer.

Despite the huge amount of visualization materials available when teaching mathematics, there is still no clear opinion on the mechanisms and degree of influence of visualization on the cognitive process.

In 1976, in the study [2] V. A. Krutetskii classified students studying mathematics in three categories:

1) geometric thinkers who prefer visual thinking;

2) analytical thinkers who choose verbal-logical thinking;

3) harmonic thinkers who prefer to use a mixture of visual and verbal methods.

The review [3] is devoted to the historical experience of visualization. It examines the different meanings of the term "visualization" in mathematics, mathematical education and psychology. Despite the fact that this review focuses on the widespread belief that mathematicians prefer to think algorithmically rather than visually, the author still talks about the need to include visualization elements in the learning process.

The article [4] notes that for the $\mathrm{Z}$ generation or iGeneration (people born between the mid-1990s and mid-200os), interactive tools are an important part of education, and therefore visualization has a significant impact on the quality of understanding abstract mathematical concepts.

The lack of reliable educational theories and criteria for the optimal use of visualization in mathematical education is noted in the review of studies on the use of visualization in 
mathematics [5]. It was concluded that empirical researches in this direction are concentrated on two main areas of mathematical education:

1) the use of visualization to promote mathematical understanding;

2) the use of visualization to assist in the construction of mathematical reasoning or in the process of computing.

Other reviews on visualization in mathematical education (for example, [6-7]) also note the positive aspects of the use of computer visualization, but at the same time they leave aside a number of methodological issues related to the visualization which require close attention.

However, the uncertainty in the theoretical study did not become an obstacle to the numerous practical implementations of visualization elements in mathematical education. For example, the positive results of the experience of similar work on the topic "Square Root" can be found in the article [8]. The experience of using visualization in teaching mathematical analysis is given in [9]. The review article [10] considers examples of computer visualization of graph algorithms. In [11], a variant of visualization of curves of second order passing through imaginary points and touching imaginary lines is proposed. The study [12] talks about the positive impact of the use of geometric visualization, which contributes to increasing interest of students to the subject. One can get acquainted with the experience of object visualization for the group theory in articles [13] and [14].

We believe that the use of visualization in the presentation of mathematical theories is an efficient tool that helps to create a single, versatile mathematical picture. Of course, the effectiveness of visualization is mostly subjective. Therefore, the naive verification (verification of correctness) based on the statistical processing of the results of the use of training materials was chosen as the methodological base of our study. The issue of verification of computer visualization is being actively investigated. Thus, for example, the article [15] is devoted to problems in this domain of scientific visualization.

We adhere to the opinion that one of the criteria for the quality of educational visualization is the insight. The importance of the insight in computer visualization is emphasized in article [16]. We believe that in order to achieve such a result as the insight, it is necessary to maintain a balance between information content (visualization) and redundancy (expressiveness) of visualization. In [17], Dwyer notes that it is difficult for students to identify the main thing if the created model contains too many details. At the same time, it is very important to minimize the user's efforts to interpret visualization; clear instructions on the use and interpretation of visualization objects are necessary. Let us remember the words of the German teacher and methodologist Adolf Disterweg: "It is more useful to consider the same issue from different sides than ten questions from one side" [18].

We believe that specific visual images can be applied when teaching many mathematical disciplines. This article discusses the issues of computer visualization when teaching $\mathrm{Ab}$ stract Algebra to future teachers of mathematics and computer science, as well as students of the training program "Mathematics and Computer Science" (undergraduate level).

The course of abstract algebra chosen for research is necessary for mathematics students for the correct perception of modern scientific ideas in the field of algebra. However, this course is the most difficult to understand due to the large number of abstract constructions, which many teachers and authors of articles note (see, for example, [19]).

The purpose of the discipline is the formation by the student' ideas about the basic algebraic structures, their place and application in various branches of mathematics, which is achieved through the objectives of the course:

1) to give knowledge of the definitions of basic algebraic structures and their most important properties;

2) to teach to work with algebraic objects;

3) to instill skills of modern algebraic thinking;

4) to introduce main directions of algebra development; 
5) to reveal connections of the course of algebra with arithmetics and the school course of mathematics;

6) to educate modern algebraic thinking.

We will consider computer visualization, since we believe it is important to teach students to actively use the computer in solving practical and theoretical problems. According to the authors of the study [20], the use of information and communication technologies (ICT) contributes to the growth of computational and algorithmic culture of students, develops spatial thinking and graphic culture, expands the range of cognitive patterns in thinking processes (perception, understanding, presentation, etc.).

We believe that it is necessary to interchange visual and non-visual ways of presenting information. The study [9] concluded that the visualization cannot be the end in itself.

Based on the arguments given above to include visualization in teaching "Abstract algebra", we consider it optimal to use the following tools to organize training as a whole:

I. The use of automation of some stages of solving tasks with visualization of intermediate results (within the framework of scientific-research work of students).

Automation of some stages of solving allows students to concentrate on the essence of the solution, and visualization of intermediate results makes it possible to better understand the decision process. The author of [21] gives the following scheme to use the visualization mechanism to find solutions of scientific tasks: "look, think, write, look, think, write, etc.". The use of computer mathematics systems in solving tasks contributes to the growth of motivation in scientific researches and the professional development of the future teacher.

II. Visualization of concepts and facts (in presentations developed for the lectures).

The use of visualization when studying abstract concepts helps students to understand better the material, encourages the search for interpretations, essential properties and relationships. The study [22] noted that the visual image is an essential factor for creating a sense of self-evidence.

III. The use of visualization in interactive applications (at the stages of the hypothesis, self-testing and when presenting the results of self-testing ).

Working with interactive models of tasks based on visual associations helps students to understand better the task and see cause-effect relations. The use of visualization at the stage of self-testing helps to strengthen the knowledge. Moreover, in the article [23], visualization is proposed to be used both in the discovery of new results and relationships, and in the process of reasoning.

Further ways for the technical implementation of each of these points are suggested. In order to develop visualization tools in the educational process, the article proposes unique solutions, algorithms for creating high-quality educational visualization with minimal time costs. Examples of using the GeoGebra system to create visualization components and to introde created objects into educational presentations using the LaTex computer document preparation system are given. To create the course website and include interactive elements in it, the use of Javascript and PHP programming languages, MySQL database and APACHE WEB server is described.

The ideas proposed in the article partially have something in common with the experience of other researchers. For example, the Department of Applied Mathematics of the University of Belgrade organized a training program "Visual Presentation of Some Mathematical Topics Using the Computer" (http://vizuelizacija.etf.rs/) in the 2010-2014 academic years for mathematics teachers and the optional course "Computer Instruments in mathematics" (http://raum.etf.rs/seminarski.html) for third-year students. In [24], the experience of the teachers of this university on the use of visualization in mathematical education using the GeoGebra and Java applications is presented.

Another example of development of visualization mechanisms is the development of a distance learning course "Fundamentals of Scientific Visualization" at NRNU MEPhI and its branches [25]. This course is aimed at studying the analysis of scientific data by the visualiza- 
tion method and is accompanied by 3ds Max and HyperFun software products, as well as the Maxscript language.

\section{Visualization of math data}

Let us demonstrate through examples the technology to implement various areas of visualization which were proposed in the introduction. results

I. Use of automation of some stages of solving tasks with visualization of intermediate

When solving mathematical tasks (including educational ones), the computer is used more and more actively. Thus, the article [26] describes the method to use computer calculations with the help of GAP to formulate mathematical hypotheses. The experience of using the Maxima computer algebra system to solve number theory tasks is described in [27]. The work with such classical objects of algebra as matrices, vector spaces, polynomials, groups, rings, fields is perfectly done in the Sage computer algebra system. The experience of using Sage in the educational process is described, for example, in the work [28].

In the following example, GeoGebra dynamic geometry system is chosen as the visualization tool. The capabilities of GeoGebra and the experience of its use in the educational process can be found in publications [29-31]. Ideas of using GeoGebra environment in the organization of student research activities are given in articles [32-33].

GeoGebra dynamic drawings created for visualization can be demonstrated in the application itself, on the geogebra.org program website and on the SMART Notebook interactive whiteboard page. All the features of the drawing developed in GeoGebra will be reflected in the iBooks e-book created by iBooks Author. It should be noted that the possibility to manipulate GeoGebra drawing objects is found when it is placed in an html document. Among the services of quick creation of WEB-pages with the possibility to insert drawings of GeoGebra, we note such site designers as Google sites, Blogger.com, Google class, services of creation distance learning environments Moodle and Math-Bridge. At the same time, the placement of the GeoGebra drawing on the html page is carried out in one of the two ways:

- insertion of the drawing exported (downloaded) in html format;

- insertion of the link to the drawing placed in the public domain through the personal account on GeoGebra website.

The following is an example of drawings that allow you to visualize the process of solving of a number of tasks.

Example 1. Let us visualize elements of the group of self-coincidences for an equilateral triangle.

To solve the task in GeoGebra, the commands Rotate (<Object $>$, < Angle $>$ ), Flip $(<$ Object $>$, $<$ Direct $>$ ), Move $(<$ Object $>$, $<$ Vector $>$ ) are enough. Fig. 1 shows a dynamic drawing in which with the changes in the vertices of the equilateral triangle $A B C$ its images are updated, which are drawn on the GeoGebra canvas below. 


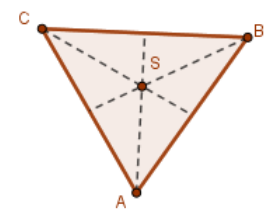

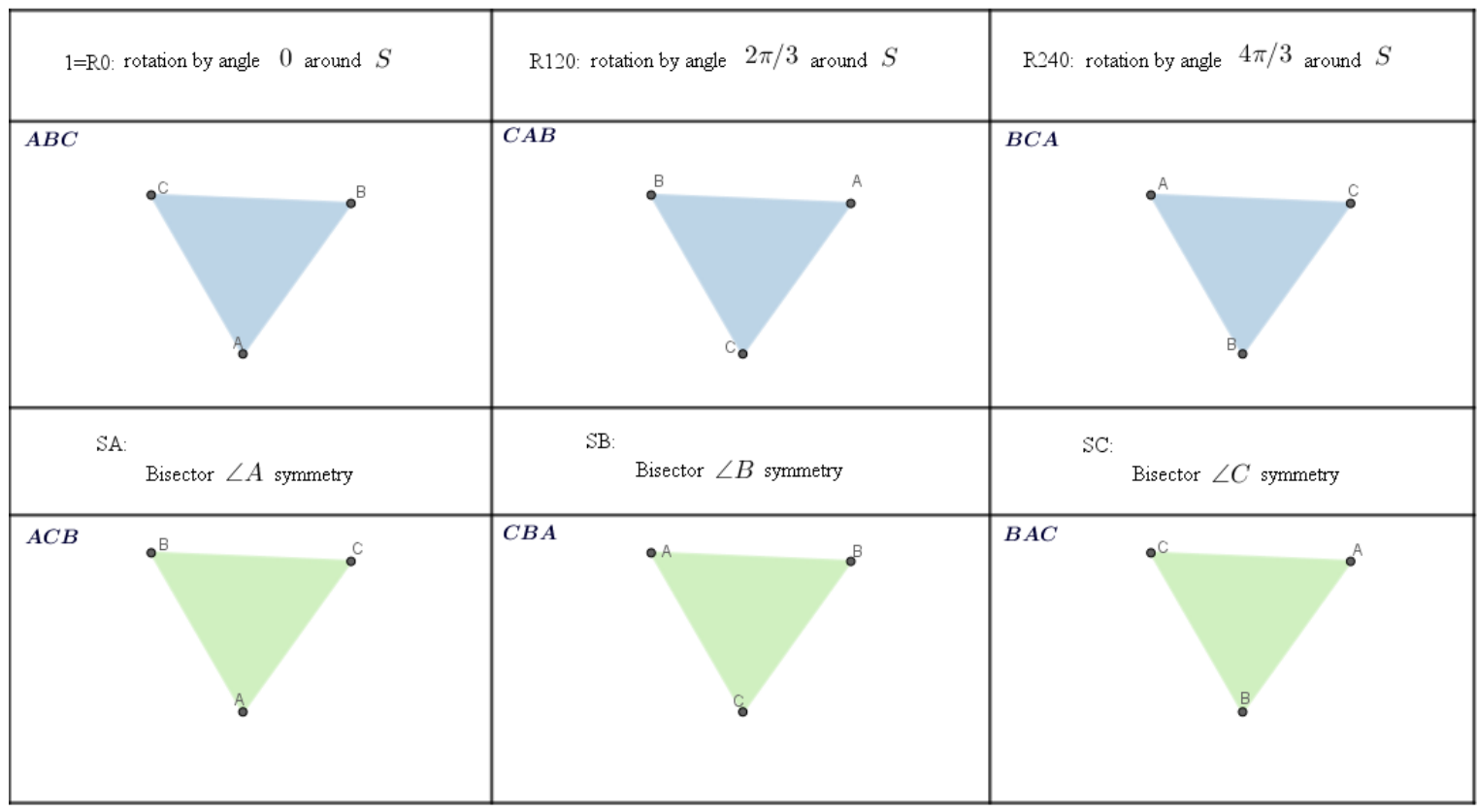

Fig. 1. Screenshot of the dynamic drawing for example 1

When creating the specified drawing, vertex images were set with short commands. For example, the image of the point A when rotating by $120 \square$ is defined by the command:

Move (Rotate (A, 120, S), (-2, -2)).

To specify the group itself, the Cayley table must be filled in for the composition operation *:

$f^{*} g$ means that at first transformation $f$ is done, then $g$.

To search for compositions of self-coincidences, it is convenient to create $\mathrm{ABC}$ triangle transformation tools using the standard GeoGebra tools :

- axial symmetries SA, SB, SC with respect to the bisectors of the angles of the triangle with subsequent transfer to a fixed vector

- rotations R120, R240 by angles $120^{\circ}$ or $240^{\circ}$ around the center of the triangle with subsequent transfer to a fixed vector.

Using the created tools you can find all possible compositions of self-coincidences. For example, as a result of the composition R120 and SB we get SC (see Fig. 2).

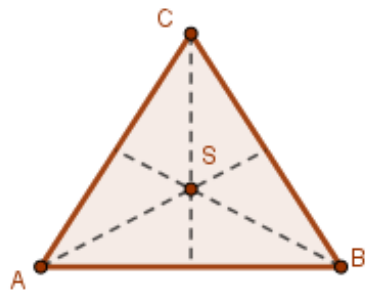
Fig. 2. Constructing the composition of self-coincidences

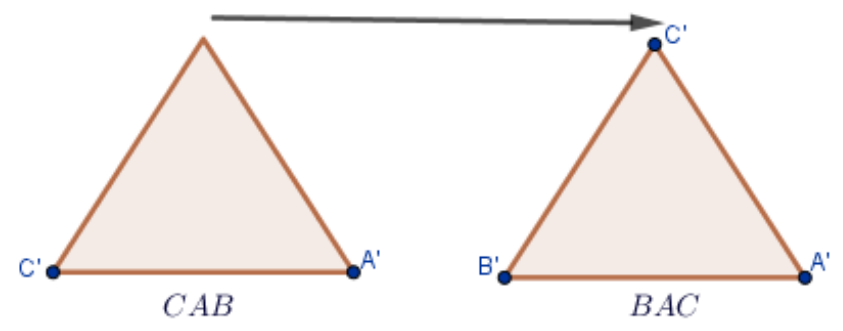


As a result, it's easy to fill in the Cayley table:

\begin{tabular}{|l|l|l|l|l|l|l|}
\hline \multicolumn{1}{|c|}{${ }^{*}$} & R0 & R120 & R240 & SA & SB & SC \\
\hline R0 & R0 & R120 & R240 & SA & SB & SC \\
\hline R120 & R120 & R240 & R0 & SB & SC & SA \\
\hline R240 & R240 & R0 & R120 & SC & SA & SB \\
\hline SA & SA & SC & SB & R0 & R120 & R240 \\
\hline SB & SB & SA & SC & R240 & R0 & R120 \\
\hline SC & SC & SA & SB & R120 & R240 & R0 \\
\hline
\end{tabular}

From the constructed table we see that this group is not commutative.

It is also useful to describe groups of self-coincindences for a square, a non-square rhombus, a rectangle with different adjacent sides and a cube. The study of some groups can be given as independent work.

II. Creating multimedia elements as illustrations for the given course

The creation of intuitively clear visual images of abstract objects using the computer allows not only to make the presented material more understandable, but also to demonstrate to future teachers the effectiveness of using ICT in teaching.

The article [34] provides various examples of creating LaTex illustrations for mathematical tasks. The following open-source animation creation tools were identified in [4]: GeoGebra, GIFsmos, GNU Octave, Julia, Matplotlib, Sage, WolframMathematica, LaTex. We offer the option of creating animations using the GeoGebra dynamic drawing system, and we will use LaTex for final preparation of the presentation in PDF format and insertion of the finished animation into it. It should be noted that in order to save time for creating a presentation pattern (or other document), you can use ready-made TeX-blanks posted on the Internet.

The mechanism Rich Media Annotation, which appeared in the 9th version of Adobe Acrobat/Reader, allows you to embed video fragments in a PDF document. The following multimedia elements can be played in such files: SWF files, video files of the MOV, MP4, $\mathrm{M}_{4} \mathrm{~V}, 3 \mathrm{GP}$ and $3 \mathrm{G} 2$ formats (if they use H.264 compression), FLV, F4V files, MP3 and MP4 audio files. However, to play files that use H.264 compression, H.264 codecs are required on the computer. In addition, the pdf document viewer must support the possibility to play videos: under Windows, you can use Adobe Reader versions 9+ for this, for Linux it can be Reader versions 9.0-9.4.1 (but not higher) or Okular.

Here is an example of creating an animated visualization, which will be further inserted into the presentation.

Example 2. Let's create an animation in GeoGebra for the theorem on homomorphism of grours.

Fig. 3 shows some frames of the corresponding animation. To set the frame change in GeoGebra, the Slider tool was used (when specifying the slider, the type was Integer, Name - a; Interval - from o to 50; in the Animation item Speed was 0.1, Action replay was selected on increase). To display different combinations of objects on different frames, the conditions for their display were set (Properties/Advanced). So, for example, when $\mathrm{a}=$ 2, only the objects shown in Fig. 3, a) should be displayed and when a = 5 we get Fig. 3, b), etc. 
a)

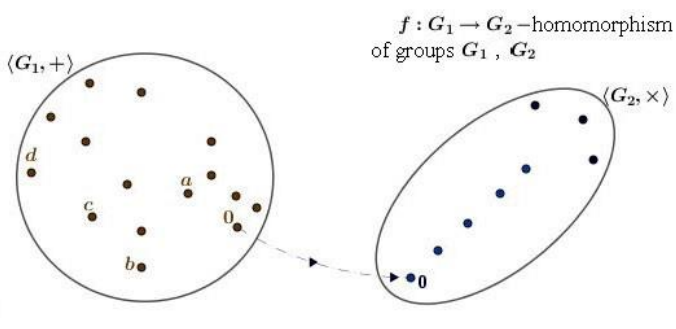

b)

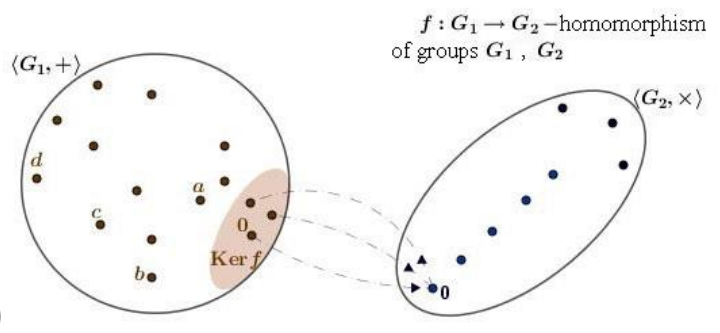

c)
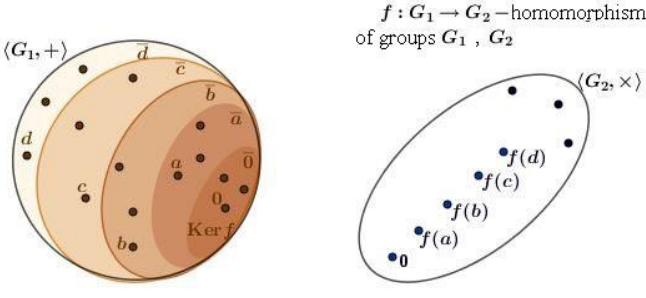

d)

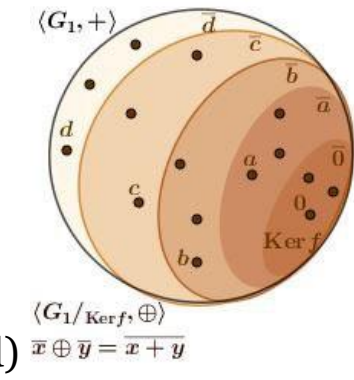

$f: G_{1} \rightarrow G_{2}-$ homomorphism of groups $G_{1}, G_{2}$

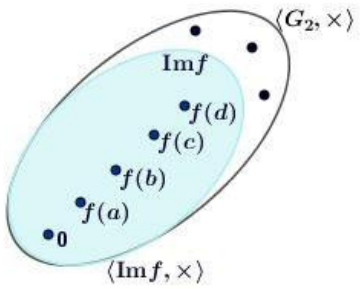

$f: G_{1} \rightarrow G_{2}$-homomorphism

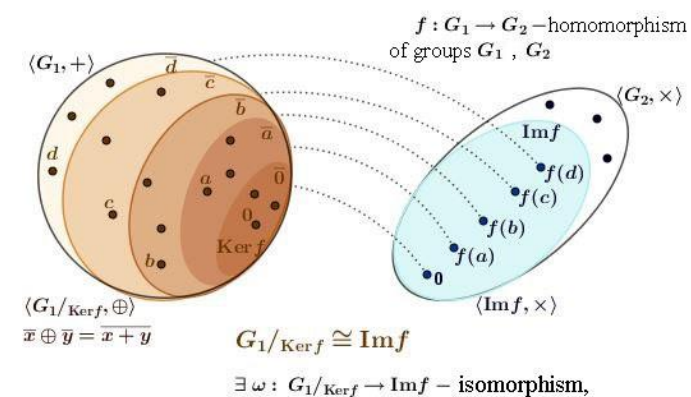

e)

1) $\omega: \bar{x} \mapsto f(x)$ - bijection

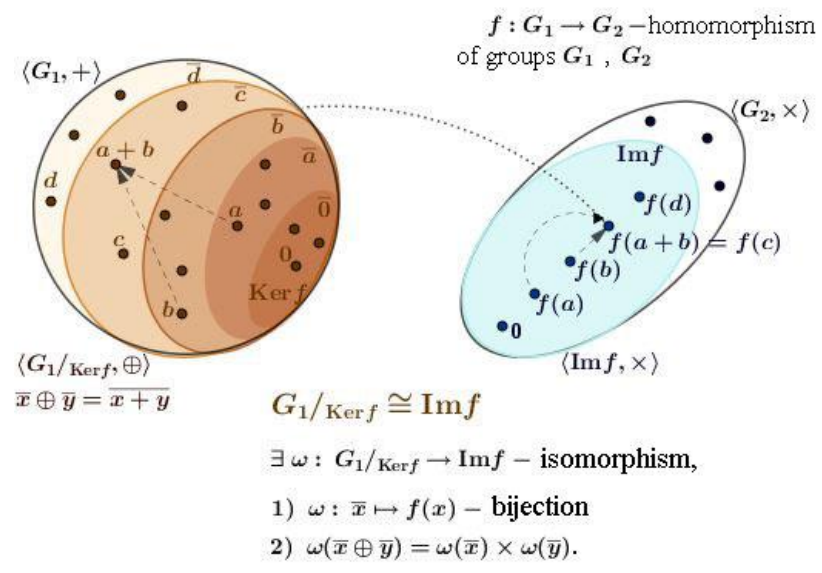

f)

$\omega(\bar{x} \oplus \bar{y})=\omega(\overline{x+y})=f(x+y)=f(x) \times f(y)=\omega(\bar{x}) \times \omega(\bar{y})$

Fig. 3. Some frames of animation for example 2

To view the designed animation of the finished drawing in GeoGebra, it is enough to select the Animate item in the slider menu, which is called on the right mouse button click. The animation can be stoped in the same way.

It should be noted that through GeoGebraTube application, you can include the dynamic drawing itself in the PowerPoint presentation. However, we consider the LaTex document preparation system to be the most suitable for creating an educational mathematical presentation. The mechanisms for including in the presentation animations and videos created in GeoGebra using the example of the discipline "Probability theory and mathematical statistics" are described in [35].

To place the created animation in a pdf presentation, you can use an image of each of the created frames separately. In this case, you can get the image of each frame (in jpg, png, pdf, etc.) using either GeoGebra tools (export to an image) or an external program that takes screen fragments. It is convenient to use the animate package to combine the resulting images into animation as a part of a pdf presentation using the TeX tools.

If the change of frames in the animation of the GeoGebra drawing depends on only one parameter, then such a drawing can be exported to a gif-animation using the program tools. Also, a running animation can be converted to a video by an external screen recording program. If necessary, the resulting file can be converted to a format acceptable for PDF. Mediag, 
a significantly optimized version of the fairly stable movie15 package, helps embed MP4, FLV, and $\mathrm{MP}_{3}$ files, as well as interactive multimedia content (SWF) files and $3 \mathrm{D}$ graphics (Adobe U3D and PRC).

We note that inserting a video into a pdf document using movie15 package requires a movie player of the appropriate format in the computer. Media9 package is independent from external video players, as it uses Flash Player built into Adobe Reader (for the latest versions). However, to insert raster video files using media9 package, you must first convert them to $\mathrm{MP} 4$ format with H.264 compression. For movie15, this is not required.

The ways of generating the final pdf document are shown in Scheme 1.

Scheme 1

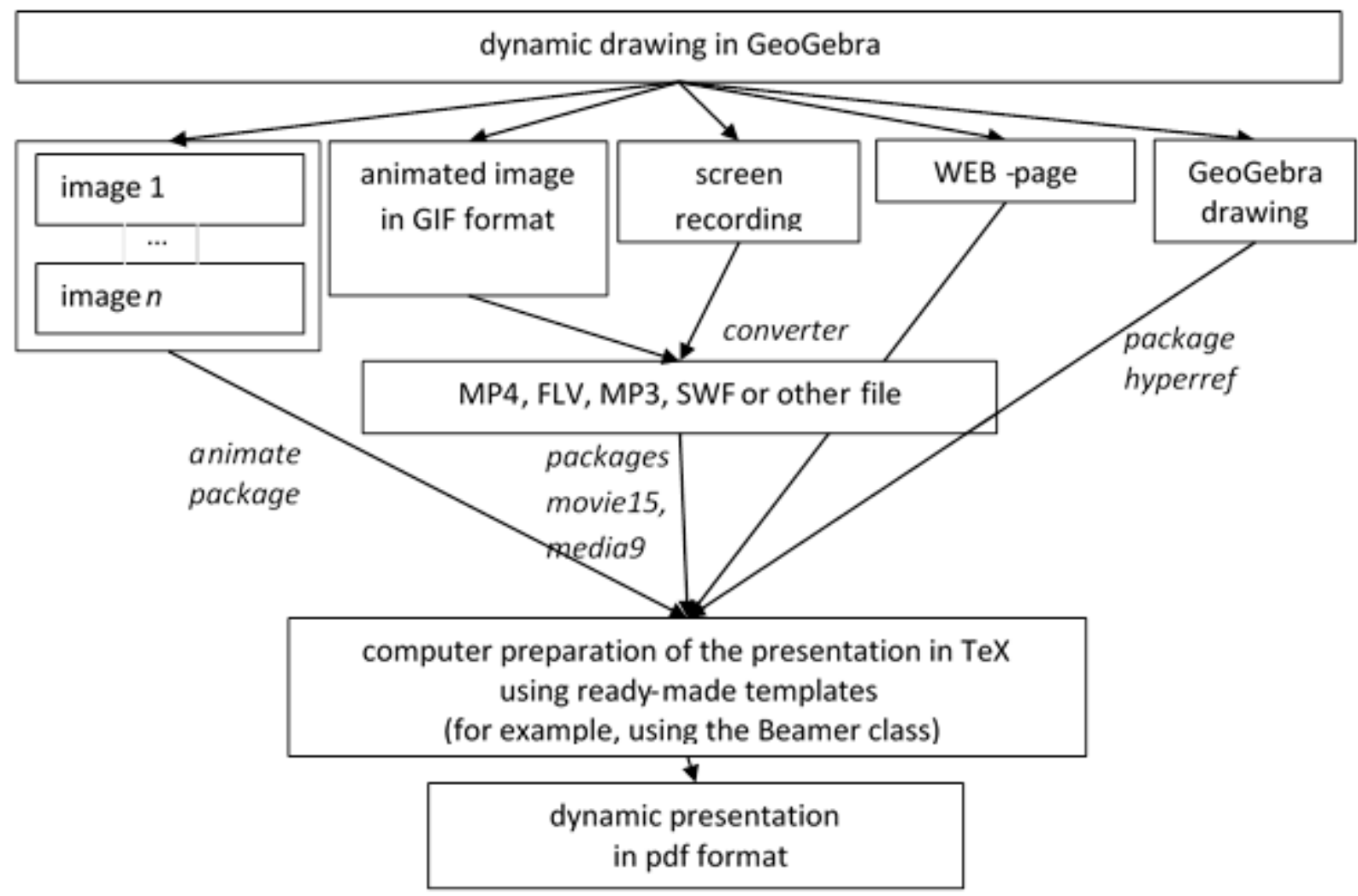

Figure 4 shows a fragment of the presentation with the visualization of the group homomorphism theorem. 


\section{The group homomorphism theorem}

The group homomorphism theorem

For any homomorphism of group G1 to group G2 there is a unique isomorphism of factor group G1 on the kernel of the homomorphism $\mathrm{f}$ on the image of group $\mathrm{G} 1$

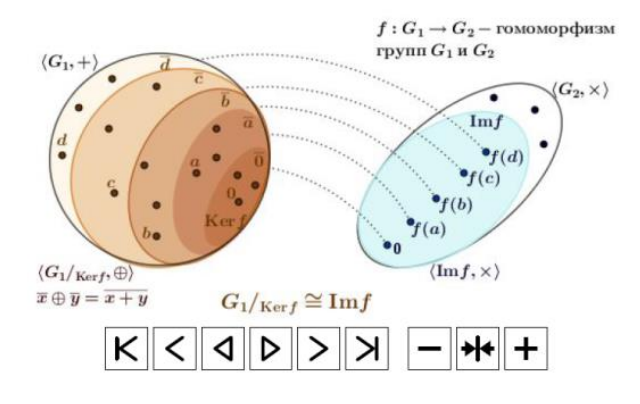

\section{Tives}

Гомоморфизмы групп. Теорема Широков Д. В.

Fig. 4. Fragment of the presentation for example 2

III. The creation of virtual laboratories and the organization of knowledge self-testing Another use of visualization in education is the virtual laboratories in cases where it is possible to vary the data. We believe that a virtual laboratory can be used to introduce the essence of the process to students, to put forward a hypothesis, and to increase the effectiveness of self-control.

In [36], the experience of the development and use of software simulators-visualizers is given. These simulators were created on the basis of the SCORM 2004 methodology, which makes it possible to collect a large number of dissimilar teaching materials into one SCORM package and use the manifest file to set the trajectory for students to pass it.

To achieve the goals of the abstract algebra course, the authors of this article designed and created an Internet site that supports interactive online tests and laboratory work, as well as their online development, administration and checking results by teachers.

The use of WEB-technologies in the development of virtual laboratories is justified by the availability of the WEB-application for all students, regardless of their software and hardware. The main advantage of the method of implementation chosen by the authors is its versatility. The implemented functionality, embedded scripts in Javascript and PHP programming languages, allows the users to interactively design, visualize and configure automatic verification of any task of the course being developed and of any task of similar courses.

Unlike conventional online testing systems, this service involves the inclusion of interactive multimedia content into the materials of test tasks, staged search of errors in the answers, and interactive tips. This approach allows to visualize the material using the extensive capabilities of the programming languages JavaScript and CSS, namely:

- display various figures, both on the plane and in space;

- movements, changes in size, color, shape of figures, etc.

- embedding interactive control elements: buttons, sliders, input fields, etc.

- wide range of possibilities of using the mathematical apparatus: testing hypotheses, properties, giving examples of dependencies, etc.

Let us indicate the software used when the site is running: access;

- PHP 5.3 for the dynamic generation of WEB-pages and organization of the data

- MySQL database for storing materials, work results and personal data of students; 
- APACHE WEB server for processing user browser requests;

- WEB browser with support of COOKIES and JavaScript to display the website data;

The main functions of the website:

- publication and editing of the materials (tests, laboratory works) with a large number of questions and answer options such as "choosing one of the options" or "text answer";

- text support of mathematical formulas typed in TEX;

- automatic checking and evaluation of the results;

- inserting images into questions and answers;

- differentiation of access rights to tests for various groups of students;

- limiting the number of attempts to do a work for one user.

In addition to the basic functions available in most online testing systems, the site has some special features: for each material on the site two display modes are available: "Test" or "Virtual Laboratory". The possibility to choose the display mode for each material is available for the teacher on the material administration page.

Unlike the Test mode, each question in the Virtual Laboratory mode is displayed on a separate page; automatic answer validation follows after each answer immediately; if the answer is incorrect, the necessary comments are given to correct the answer, and it is proposed to answer the question again, or to go to the next question. This mode is suitable for independent assimilation of educational material by students, or as informational support during practical classes.

Besides the text and images, site materials allow the insertion of scripts into PHP and Javascript programming languages.

Thanks to the placement of tasks made by the usage of scripts in Javascript language, the tasks include not only text, formulas and images, but also various interactive elements supported by Javascript. Scripts allow to embed controlled animation into the website, text input fields, data manipulators - "sliders", etc.

The main difference between PHP scripts and Javascript is that the first ones are executed on the WEB server, and the second ones are executed in the user's WEB browser. The use of PHP scripts eliminates accidental or intentional viewing of the answer checking algorithm by the testee and, as a result the selection of the correct answer without solving the task. The second feature of using the PHP script to check the text answer allows to hide the text answer field in the question containing Javascript. In this case, the Javascript of the question automatically generates the answer and passes it to the PHP script for checking the answer escaping the text field.

All pages of the site are tested for the possibility to work and do tests using mobile devices with a small screen. This eliminates the need for display classes when conducting interactive laboratory work.

Example 3. The virtual laboratory on the topic "Groups".

The virtual laboratory created for this topic is on the open Internet at http://testdip.markovrv.ru. Fig. 5 shows a fragment of it. 


\section{Virtual laboratory}

$1 2 3 \longdiv { 5 6 }$

4) The congruence motion group of the triangle. The figure shows the triangle obtained after applying the following chain of transformations: R240, SA, R120, R120, SB, SC.

Find the original triangle.

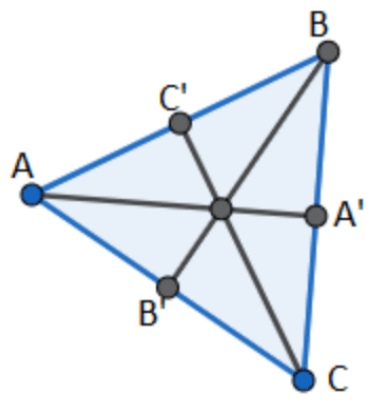

$\underline{\mathrm{R} 240} \mid \underline{\mathrm{R} 120}$

$\underline{\mathrm{SA}}|\underline{\mathrm{SB}}| \underline{\mathrm{SC}}$

Fig. 5. Display of study materials in the mode "Virtual Laboratory"

Interactivity of visualization in the above example is provided by the JavaScript code embedded in its text:

var pos = 'abc';

var el = \%qID\%;

var $\mathrm{p}=$ el+'_pic';

var link = 'pic/';

sethtml(el, '<img width="20opx" src="'+link+pos+'.png" id="'+p+"'><br $>$ + createA("pos=oper(pos,'pp',"'+p+"')",'R240') + " | " +

createA("pos=oper(pos,'pl','"+p+"')",'R120') + "<br>"+

createA("pos=oper(pos,'oa',"'+p+"')",'SA') + " | " +

createA("pos=oper(pos,'ob','"+p+"')",'SB') + " | " +

createA("pos=oper(pos,'oc','"+p+"')",'SC')

)

Here sethtml (), oper () and create () are user-defined functions ${ }^{1}$, that are responsible for displaying and moving the tested shape.

If the answer is incorrect, the user receives, in addition to the message on the incorrect answer, a prompt message on how to correct the solution (see Fig. 6). The prompt text is fixed when writing the text of the question, but it can be changed by the PHP script of checking the answer.

\footnotetext{
${ }^{1}$ The code of these functions is available at the link http://testdip.markovrv.ru/my.js
} 


\section{Virtual laboratory}

$1 2 \longdiv { 3 4 5 6 }$

2) Define the operation * on the set $\{a, b\}$ so that to get the semigroup

\begin{tabular}{|c|c|c|}
\hline * & $\mathbf{a}$ & b \\
\hline a & 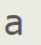 & $a$ \\
\hline & b & $a$ \\
\hline
\end{tabular}

The answer is incorrect

Prompt: the condition $(b * a) * b=b *(a * b)$ is not met in your solution

Repeat

Next

Exit

Fig. 6. Display of a prompt if the answer is incorrect

Let us give an example of such a script as applied to the task presented in Fig. 6:

if(strlen $(\$$ val $)<4$ || !preg_match("/(a|b)(a|b)(a|b)(a|b)/",\$val))\{

$\$$ res $=0$;

$\$$ help=" The cells must contain only the elements a and b.“.

" All cells must be filled."; $\$ \operatorname{val}\{3\}$;

\}else\{

$\$ \mathrm{~m}\left[\mathrm{a}^{\prime}\right]\left[\mathrm{a}^{\prime}\right]=\$ \operatorname{val}\{\mathrm{o}\} ; \$ \mathrm{~m}\left[\mathrm{a}^{\prime}\right]\left[\mathrm{b}^{\prime}\right]=\$ \operatorname{val}\{1\} ; \$ \mathrm{~m}\left[\mathrm{~b} \mathrm{~b}^{\prime}\right]\left[\mathrm{a}^{\prime}\right]=\$ \mathrm{val}\{2\} ; \$ \mathrm{~m}\left[\mathrm{~b}^{\prime}\right]\left[\mathrm{b}^{\prime}\right]=$

$\$ \mathrm{cnt}=0$;

if $\left(\$ m\left[\$ m[' a ']\left[a^{\prime}\right]\right]\left[' a^{\prime}\right]==\$ m\left[{ }^{\prime} a^{\prime}\right]\left[\$ m\left[{ }^{\prime} a^{\prime}\right]\left[{ }^{\prime} a^{\prime}\right]\right]\right) \$ c n t++$;

else $\$$ help $=$ " Your solution does not satisfy the condition $\backslash \$\left(a^{*} a\right)^{*} a=a^{*}\left(a^{*} a\right) \backslash \$ "$;

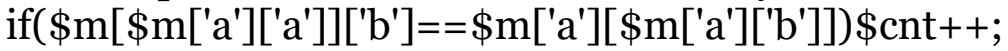

else $\$$ help $=$ " Your solution does not satisfy the condition $\backslash \$\left(a^{*} a\right)^{*} b=a^{*}(a * b) \backslash \$ " ;$

$/ /$ the rest conditions are described similarly

if $(\$ c n t==8) \$ r e s=1 ;$ else $\$ r e s=0 ;\}$

Example 4. Visualization of students' achievements.

It was possible to visualize the results of work in the form of diagrams using the GoogleChart ${ }^{2}$ service. Fig. 7 presents a variant of the diagram, which is available to each student in his/her personal account. This service allows the student to evaluate his/her success when working on the site. 


\section{Welcome, Ivanov Petr Semyonovich}

\section{Diagram of grades}

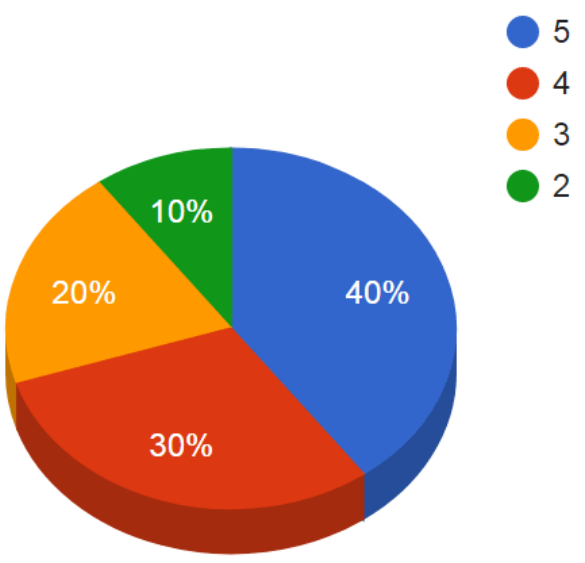

Fig. 7. Diagram "Student's grades"

Figure 8 shows an example of the diagram "Grades of the group", which is available for the teacher on the page of the results of the work. It allows the teacher to evaluate the success of a group of students who has done the tasks.

\section{The list of students' grades}

Demonstration group

of those who took part in testing

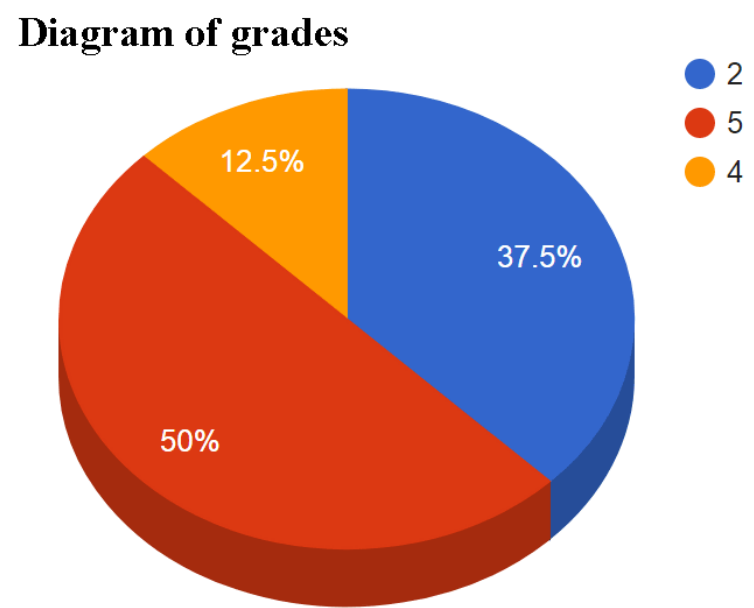

Fig. 8. Diagram "Grades of the group"

An example of the diagram "Distribution of answers" is presented in Fig. 9. It is available for the teacher and allows him to analyze the students' answers to each question, which helps to plan the further work. 


\section{Report on academic progress of the group \\ Demonstration group \\ in the context of the test "Virtual laboratory"}

define a NON-commutative operation on the set $\{a, b\}$

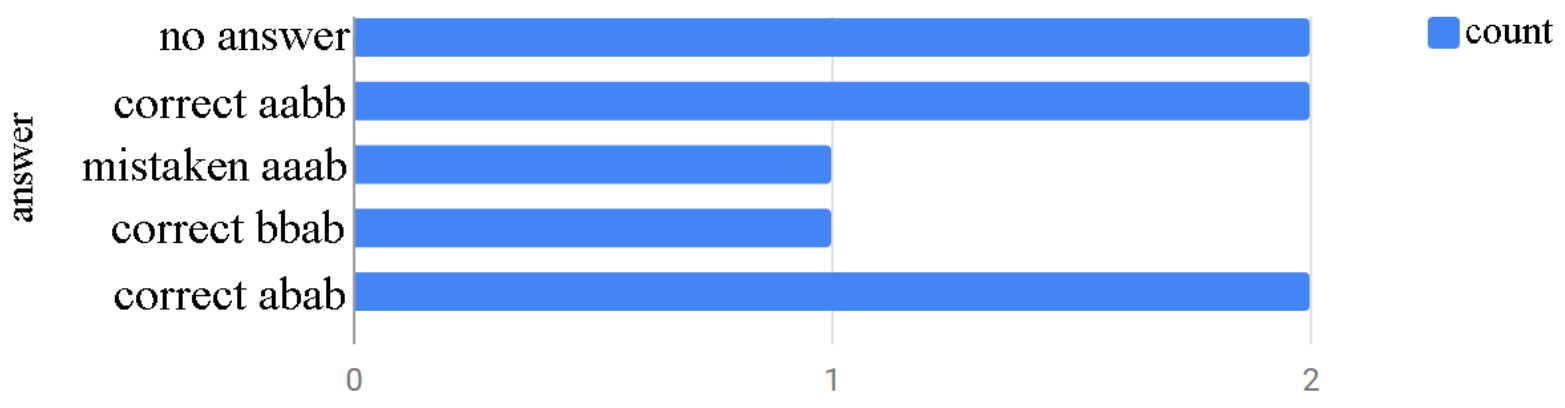

Fig. 9. Diagram "Distribution of answers"

\section{Results}

The verification of the created with the help of visualization concepts of abstract algebra was carried out by mathematical students on the basis of subjective assessments of "understandability" and "effectiveness" of the proposed illustrations. Some visualization models have been optimized using of the results of the interaction.

For experimental assessment of validity of the created visualizations and the impact of visualizations on the learning process, the level of knowledge obtained on their basis was determined. The measurement was carried out when testing students of two groups. One group (control) was trained in the usual way, lectures and practical classes in this group were held without the use of the computer support. In the second group (experimental) throughout the course, elements of visualization were used both during the lectures and tutorials.

Assessing the indicators on the 30-point scale after studying the course of abstract algebra, we note that the average score in the experimental group (23.64) was higher than the average score of the control group (18.07). In order to statistically prove that the level of knowledge of the experimental group is higher than in the control group, the nonparametric Wilcoxon-Mann-Whitney test was used [38]. The choice of this particular criterion, and not Student's criterion, is justified by the fact that in this case we do not know which distribution law the selection made obeys.

Statistical assessment of the results was carried out in two stages. During the first stage, the comparison of the differences between the indicators of the level of knowledge in algebra before taking the course in abstract algebra was made. To do this, tests on the studied algebra topics were carried out, and according to their results, the scores in the selected groups were compared. The average score (on the 20-point scale) in the experimental group was 14,64, and the average score in the control group was 14,35, while the variability of the experimental group exceeded the variability of the control group. We formulate the null hypothesis: differences in the indicators of the compared samples are random and insignificant. To verify it, we use the specified statistical criterion. Having placed all the values of the two samples in a ranked series, we calculate the empirical value of the criterion statistics: $\mathrm{U}_{\mathrm{EMP}}=79$. At the significance level of $\mathrm{a}=0,05$, we determine the critical value $\mathrm{U}_{\mathrm{CR}}=55$. Since $\mathrm{UEMP}_{\mathrm{EM}}$ U UR, we conclude that there are no grounds for the selected significance level to reject the null hypothesis, that is, we can assume that before the experiment both groups had the same level of knowledge in algebra. 
The second stage of the test consisted of comparing the test results in the two groups after conducting classes in abstract algebra. We leave the null hypothesis the same. We calculate the empirical value of the statistics of the criterion $U_{\mathrm{EMP}}=49$ and compare it with the critical value $U_{C R}=55$ at the significance level $\square=0,05$. Taking into consideration the inequality $\mathrm{UEMP}_{\mathrm{EM}}<\mathrm{U}_{\mathrm{CR}}$, we find that the test results in the experimental group are higher than in the control group. Therefore, with the probability of 0.95 it can be confirmed that the indicators of the level of knowledge of the two studied groups differ in favor of the experimental group.

Based on naive verification, we conclude that the created software and tool complex for visualizing objects and concepts of abstract algebra correctly displays their content, and its use positively affects the learning process.

The described experience of introducing visualization at different stages of training reflects the authors' view on the problem raised. The work discusses modern software and hardware capabilities for creating and implementing mathematical visualizations in the educational process. The presented service "Interactive Laboratory" is unique in the field of educational software. The service can be used to create other interactive laboratories for teaching mathematical disciplines, which is especially important due to the growing trends to introduce ICT in the education.

\section{References}

1. Zimmermann W., CunninghamS. Visualization in teaching and learning mathematics. Washington: Math. Assoc. of America. 1991. 224 p.

2. Krutetskii V. A. The psychology of mathematical abilities in school children. Chicago: University of Chicago Press. 1976. 417 p.

3. Clements M..A. Fifty Years of Thinking About Visualization and Visualizing in Mathematics Education: A Historical Overview. In: Fried M., Dreyfus T. (eds) Mathematics \& Mathematics Education: Searching for Common Ground. Advances in Mathematics Education. Springer, Dordrecht. 2014. P.177-192. DOI: 10.1007/978-94-007-7473-5_11

4. Mojžišová A., Pócsová J. Visualisation of mathematical content using LATEX animations //19th International Carpathian Control Conference (ICCC). Szilvasvarad, Hungary.2018. P. 536-541. DOI: 10.1109/CarpathianCC.2018.8399689

5. Macnab J. S., Phillips L. M., Norris S. P. Visualizations and Visualization in Mathematics Education. In: Norris S. P. (eds) Reading for Evidence and Interpreting Visualizations in Mathematics and Science Education. Sence Publishers, Rotterdam. 2012. P. 103-122.

6. Kadunz G., Yerushalmy M. Visualization in the Teaching and Learning of Mathematics In: Cho S. J. (eds) The Proceedings of the 12th International Congress on Mathematical Education. Springer, Cham. 2015. P. 463-467. DOI: 10.1007/978-3-319-12688-3_41

7. Arcavi A. The role of visual representations in the learning of mathematics // Educational Studies in Mathematics. 2003. Vol. 52(3). P. 215-241. DOI: 10.1023/A:1024312321077

8. Simmt E., Sookochoff S., McFeetors J., Mason R. T. Curriculum development to promote visualization and mathematical reasoning: radicals In: Norris S.P. (eds) Reading for Evidence and Interpreting Visualizations in Mathematics and Science Education. Sense Publishers, Rotterdam. 2012. P. 147-163. DOI: 10.1007/978-94-6091-924-4_8

9. Nardi E. Reflections on Visualization in Mathematicsand in Mathematics Education. In: Fried M., Dreyfus T. (eds) Mathematics \& Mathematics Education: Searching for Common Ground. Advances in Mathematics Education. Springer, Dordrecht. 2014. P. 193220. DOI: $10.1007 / 978-94-007-7473-5 \_12$

10. Gordeev D. S. The survey of visualization of algorithms on graphs.// Scientific Visualization. 2018. Vol. 10. No. 1. P. 18-48. DOI: 10.26583/sv.10.1.02 [In Russian]

11. Korotkiy V. A. Computer visualization of curves of second order going through the imaginary point and touching imaginary lines // Scientific Visualization. 2018. Vol. 10. No. 1. P. 56-68. DOI: 10.26583/sv.10.1.04 [In Russian] 
12. Dundara S., Gokkurt B., Soylu Y. The efficiency of visualization through geometry at mathematics education: a theoretical framework // Procedia-Social and Behavioral Sciences. V. 46. P. 2579-2583.

13. EvansT. J. Discovering Binomial Identities with PascGaloisJE // PRIMUS. 2008. Vol. 18 (4). P. 361-369.DOI: 10.1080/10511970701299492

14. Bardzell M. J., Shannon K. M. The PascGalois Project: Visualizing Abstract Algebra // MAA Focus. 2002. Vol. 22 (3). P. 4-5.

15. Manakov D., Averbukh V. Verification of visualization // Scientific Visualization, 2016, volume 8, number 1, P. 58 - 94.

16. Averbukh V.L., Averbukh N.V. On the question of insight in computer visualization // Digital Society as a cultural and historical context of human development. Collection of scientific articles and materials of the international conference, 2016, P. 12-15.

17. Dwyer F. M. When visuals are not the message. // Educational Broadcasting Review. Vol. 2 (5). 1968. P. 38-43.

18. Disterveg A. Selected pedagogical works. M.: Uchpedgiz, 1956. 378 p. [In Russian]

19. Hill J., Thron C. Elementary Abstract Algebra: Examples and Applications. 2017. 785 p. http://abstractalgebra.altervista.org/aafmt.pdf(access: 01.08.2018)

20. Smirnov E.I., Bogun V.V. Visual Modeling Using ICT in Science and Mathematics Education. In: Elleithy K., Sobh T., Mahmood A., Iskander M., Karim M. (eds)Advances in Computer, Information, and Systems Sciences, and Engineering. Springer Netherlands. 2007. P. 453-458 DOI: 10.1007/1-4020-5261-8_70

21. Davis R. B. Learning mathematics: the cognitive science approach to mathematics education. New Jersey: Ablex. 1984. 392 p.

22. Fischbein E. Intuition in science and mathematics: An educational approach. Springer Science+Business Media Dordrecht. 2002. 226 p. DOI: 10.1007/o-306-47237-6

23. Dreyfus T. Imagery and reasoning in mathematics and mathematics education. In: Robitaille D. F., Wheeler D. H., Kieran C. (eds) Selected Lectures from the 7 th International Congress on Mathematical Education. Quebec, Canada: Les Presses de l'Université Laval. 1994. P. 107-122.

24. Malešević B. J., Jovović I. V., Banjac B. D. Visualization in teaching and learning mathematics in elementary, secondary and higher education // Proceedings of International Conference on Engineering Graphics and Design. Timisoara, Romania. 2013. P.37-40.

25. Kryuchkov E., Alekseev V., Ermakov S. and others. The experience of development and testing the distance educational course "Fundamentals of scientific visualization" at NRNU "MEPHI" and its branches// Scientific Visualization. 2014. Vol. 6. No. 1. P. 20-30. [In Russian]

26. Blyth R. D. Rainbolt J. G. Discovering Theorems in Abstract Algebra Using the Software GAP // Primus.2010. Vol. 20 (3). P. 217-227. DOI: 10.1080/10511970802119714

27. Sirazov F. S. Use of Maxima Computer Algebra System in Teaching Elements of Abstract and Computer Algebra // News of RGPU named after A.I. Gertsen. 2009. No. 116. P. 223-227. [In Russian]

28. Yatskin N.I. Algebraic calculations in Sage system. Ivanovo: Ivanovo State University. 2014. 47 p. [In Russian]

29. Shirikova T. S. The method of teaching students of the basic school to prove theorems when studying geometry using GeoGebra. Thesis for the degree of Candidate of Pedagogical Sciences. Arkhangelsk. 2014. 250 p. [In Russian]

30. Bezumova O. L., Ovchinnikova P. R., Troitskaya O. N. and others. Teaching Geometry using GeoGebra capabilities: a study guide. Arkhangelsk: KIRA. 2011. 140 p. [In Russian]

31. Larin S. V. Computer animation in the GeoGebra environment at the lessons of mathematics. Rostov-on-Don: The Legion. 2015. 192 p. [In Russian]

32. Lubyagina E. N, Timshina L. V, Shirokov D. V. Visualization of regular $n$ dimensional polyhedra // Advanced Science. 2018. No. 1. P. 13-20. [In Russian] 
33. Lubyagina E. N., Timshina L. V. Experience in the organization of students' educational and research activities when studying curves of second order // Bulletin of Syktyvkar University. Ser. 1: Mathematics. Mechanics. Computer sciences. 2017. Vol. 2 (23). P. $70-84$. [In Russian]

34. Kaneko M., Abe T., Fukazawa K. and others. CAS-aided Visualization in LATEX documents for Mathematical Education // Teaching Mathematics and Computer Science. Vol. 8. P. 1-18.DOI: 10.5485/TMCS.2010.0172

35. Lubyagina E. N., Timshina L. V., Shirokov D. V. Visual support of the course "Theory of Probability and Mathematical Statistics" // Science and Innovations in the 21st Century: Current Issues, Discoveries and Achievements. Collection of articles of the V International Scientific and Practical Conference. 2017. P. 12-21. [In Russian]

36. Guseva A. I., Kireev V. S., Kozhin I. M. and others. Visualization of objects in software simulators - multi-step solvers in the format SCORM 2004 // Scientific visualization. 2013. Vol. 5. No. 2. P. 34-52. [In Russian]

37. Neuhäuser M. Wilcoxon-Mann-Whitney Test // International Encyclopedia of Statistical Science. Berlin, Heidelberg: Springer Berlin Heidelberg. 2011. P. 1656-1658. DOI: 10.1007/978-3-642-04898-2_615

38. Neuhäuser M. Wilcoxon-Mann-Whitney Test // International Encyclopedia of Statistical Science. Berlin, Heidelberg: Springer Berlin Heidelberg. 2011. P. 1656-1658. DOI: 10.1007/978-3-642-04898-2_615 